\title{
Optimized perturbation methods for the free energy of the anharmonic oscillator
}

\author{
Kostas Vlachos \\ Department of Physics, Patras University \\ GR 26110 Patras, Greece \\ Anna Okopińska \\ Institute of Physics, Warsaw University, Białystok Branch, \\ Lipowa 41, 15-424 Białystok, Poland \\ e-mail: rozynek@fuw.edu.pl
}

November 21, 2018

\begin{abstract}
Two possibile applications of the optimized expansion for the free energy of the quantum-mechanical anharmonic oscillator are discussed. The first method is for the finite temperature effective potential; the second one, for the classical effective potential. The results of both methods show a quick convergence and agree well with the exact free energy in the whole range of temperatures.
\end{abstract}


1. The quantum mechanical anharmonic oscillator $(\mathrm{AO})$ with a Hamiltonian

$$
H=\frac{p^{2}}{2}+\frac{m^{2} x^{2}}{2}+\lambda x^{4}
$$

is equivalent [1] to the theory of a scalar field in the Euclidean space-time of one-dimension (time) with a classical action given by

$$
S[x]=\int\left[\frac{1}{2} x(t)\left(-\partial^{2}+m^{2}\right) x(t)+\lambda x^{4}(t)\right] d t .
$$

After rescaling all quantities in terms of $\lambda$, only one dimensionless parameter $z=\frac{m^{2}}{\lambda^{2 / 3}}$ remains; therefore, when discussing numerical results, we put $\lambda=1$ without a loss of generality. The energy spectrum of the AO can be calculated numerically and provides a simplest test for approximation methods in quantum field theory (QFT) (f.e. loop expansion [2], 1/N expansion, loop expansion for composite operators [3], optimized expansion (OE) [4]). The conventional loop expansion gives the Rayleigh-Schrödinger series which is asymptotic for the AO [1]; hence, the numerical results for energy levels are good only for large values of the parameter $\frac{m^{2}}{\lambda^{2 / 3}}$. In the $1 / N$ expansion the energy levels can be calculated from spectral properties of Green's functions, derived from the given order effective action. The results show a quick convergence and agree well with the exact spectrum of the AO, the quality of approximation decreases for increasing level of excitation [5]. This is similar in the OE; however, in this case the approximation becomes worse as the parameter $\frac{m^{2}}{\lambda^{2 / 3}}$ decreases, becoming unapplicable in the double-well case $\left(m^{2}<0\right)$ [5].

It is also interesting to discuss the partition function and the free energy which contain information on the whole spectrum of the quantum system. The field-theoretical methods for systematic approximations of the effective action can be extended to finite temperature $T$ in the Euclidean formalism by compactifying the "imaginary time" dimension with a period $\beta=\frac{1}{T}$. The conventional loop expansion coincides with termodynamic perturbation theory for free energy [6]. Here we study the approximations for the free energy, obtained by two applications of the OE: (i) for the finite temperature effective potential, (ii) for the classical effective potential.

2. The first method is an extension of the $\mathrm{OE}$ for the effective action in QFT [4] to finite temperature effective potential and has been studied in the 
space-time of arbitrary dimension [7]. Here we discuss the one-dimensional case when the vacuum persistence amplitude is defined by the integral

$$
Z[J]=\int D x e^{-S[x]+\int J(t) x(t) d t},
$$

over the functions $x(t)$ which vanish at infinity. The effective action is defined by

$$
\Gamma[\xi]=\ln Z[J]-\int \xi(t) J(t) d t
$$

where the expectation value of $x$ is given by $\xi(t)=\frac{\delta \ln Z}{\delta J(t)}$. The extension to finite temperature $T$ consists in replacing the set of functions in the integral (3) by periodic functions with a period $\beta=\frac{1}{T}$. The infinite interval in all integrals over $t$ has to be replaced by the interval $[0, \beta]$, we denote the corresponding quantities by $Z_{\beta}, \Gamma_{\beta}$. The finite temperature effective potential defined by

$$
V_{\beta}(\xi)=\left.\frac{1}{\beta} \Gamma_{\beta}[\xi]\right|_{\xi(t)=\xi}
$$

has a meaning of the free energy of the quantum system interacting with a constant electric field $J$, when the expectation value of the coordinate $x$ is given by $\xi=$ const. The free energy $F$ of the $\mathrm{AO}$ is determined by the value of the finite temperature effective potential at minimum which corresponds to $J=0$.

The $\mathrm{OE}$ for the effective action is generated by the application of the steepest-descent method to the generating functional $Z[J]$ with the classical action modified to the form

$$
\begin{aligned}
S_{\epsilon}[x] & =\int \frac{1}{2} x(t) G^{-1}\left(t, t^{\prime}\right) x\left(t^{\prime}\right) d t d t^{\prime} \\
& +\epsilon\left[\int \frac{1}{2} x(t)\left(D^{-1}\left(t, t^{\prime}\right)-G^{-1}\left(t, t^{\prime}\right)\right) x\left(t^{\prime}\right) d t d t^{\prime}+\lambda \int x^{4}(t) d t\right],
\end{aligned}
$$

where $D^{-1}\left(t, t^{\prime}\right)=\left(-\partial^{2}+m^{2}\right) \delta\left(t-t^{\prime}\right)$. After shifting $x(t)$ by $x_{0}(t)$ chosen to satisfy the classical equation of motion $\frac{\delta S_{\epsilon}}{\delta x_{0}(t)}=-J(t)$ and expanding the exponential into Taylor series we obtain 


$$
\begin{aligned}
Z[J] & =e^{-S_{\epsilon}\left[x_{0}\right]+\int J(t) x_{0}(t) d t} \int D x^{\prime} e^{-\int \frac{1}{2} x^{\prime}(t) G^{-1}\left(t, t^{\prime}\right) x^{\prime}(t) d t d t^{\prime}} \\
\times[1 & -\epsilon\left(\int \frac{1}{2} x^{\prime}(t)\left(\Delta^{-1}\left(t, t^{\prime}\right)-G^{-1}\left(t, t^{\prime}\right)\right) x^{\prime}\left(t^{\prime}\right) d t d t^{\prime}+\lambda \int x_{0}(t) x^{\prime 3}(t) d t\right. \\
& \left.\left.+\lambda \int x^{4}(t) d t\right)+\ldots\right],
\end{aligned}
$$

where $\Delta^{-1}\left(t, t^{\prime}\right)=\left(-\partial^{2}+m^{2}+12 \lambda x_{0}^{2}(t)\right) \delta\left(t-t^{\prime}\right)$. Upon Legendre transform, the effective action (4) is obtained as a series in $\epsilon$. The $n$th order term can be represented diagramatically as a sum of $n$-vertex vacuum one-particleirreducible diagrams with Feynman rules of the modified theory (6). The third order result is shown in Fig.1.

3. The finite temperature effective potential in the $\mathrm{OE}$ is given by the same set of diagrams as the effective action, only the Feynman rules are replaced by those at finite temperature. Since $V_{\beta}$ is a function of constant $\xi$, the propagator can be chosen in the form

$$
G_{\beta}\left(t, t^{\prime}\right)=\frac{1}{\beta} \Sigma_{m=-\infty}^{\infty} \exp \left[-i \omega_{m}\left(t-t^{\prime}\right)\right] \frac{1}{\omega_{m}^{2}+\Omega^{2}}=\frac{\cosh \left[\frac{\Omega}{2}\left(\left|t-t^{\prime}\right|-\beta\right)\right]}{\Omega \sinh \left[\frac{\beta \Omega}{2}\right]},
$$

with an arbitrary parameter $\Omega$. The Matsubara frequencies are given by $\omega_{m}=\frac{2 \pi m}{\beta}$. At zero temperature the propagator becomes equal to

$$
G_{\infty}\left(t, t^{\prime}\right)=\int \frac{d p}{2 \pi} \exp \left[-i p\left(t-t^{\prime}\right)\right] \frac{1}{p^{2}+\Omega^{2}}=\frac{\exp \left[-\Omega\left(\left|t-t^{\prime}\right|\right]\right.}{2 \Omega} .
$$

The parameter $\epsilon$ is a formal parameter of expansion and is set equal to one at the end. The exact $V_{\beta}(\xi)$, obtained as a sum of an infinite series, does not depend on $\Omega$, but a finite order truncation does. We can make the $n$ th-order approximant $V_{\beta}^{(n)}(\Omega, \xi)$ as insensitive as possible to small variation of the unphysical parameter by choosing $\Omega$ equal to $\tilde{\Omega}(n, \xi, \beta)$ satisfying the gap equation

$$
\frac{\delta V_{\beta}^{(n)}}{\delta \tilde{\Omega}}=0
$$

In the approximate expression for the finite temperature effective potential $V_{\beta}^{(n)}(\xi)=V_{\beta}^{(n)}(\xi, \tilde{\Omega}(n, \xi, \beta))$ the optimal value of $\Omega$ changes from order to 
order, assuring the convergence of the expansion [8]. The first order of the OE coincides with the finite temperature Hartree approximation, but the variational interpretation cannot by maintained beyond the first order.

We calculated the finite temperature effective potential to third order in the OE. In second order the gap equation has no real solution in some range of temperatures, in this case the real part of the result has been taken. For the single-well AO $\left(m^{2}>0\right)$ the symmetric minimum of the finite temperature effective potential at $\xi=0$ (OES) gives a very good description of the free energy. The quality of approximation becomes worse for decreasing $\frac{m^{2}}{\lambda^{2 / 3}}$. In the most unfavourable case of the quartic oscillator $\left(m^{2}=0\right)$ the results of three lowest orders are shown in Fig. 2 in comparison with the exact free energy, calculated by the numerical procedure based on the modification of the linear variational method 9 .

In the double-well case $\left(m^{2}<0\right)$, there is a critical value $z_{c r}(n, \beta)$ of the parameter $\frac{m^{2}}{\lambda^{2 / 3}}$, above which the only minimum of $V_{\beta}^{(n)}(\xi)$ is at $\xi=0$, in agreement with the exact result. However, for $\frac{m^{2}}{\lambda^{2 / 3}}$ below $z_{c r}$ a lower minimum at $\xi \neq 0$ appears. As can be seen in Fig. 3, for $m^{2}=-20$, the value of the finite temperature effective potential in the non-symmetric minimum $(\mathrm{OEN})$ gives much better approximation of the free energy than the value at $\xi=0(\mathrm{OES})$.

4. Different approximation schemes for the free energy can be obtained [10] approximating the classical effective potential $V_{c l}(x)$, defined by a simple integral

$$
Z_{\beta}[J=0]=e^{-\beta F}=\int \frac{d x_{0}}{\sqrt{(} 2 \pi \beta)} e^{-\beta V_{c l}\left(x_{0}\right)} .
$$

The local partition function $Z^{x_{0}}$ can be written as

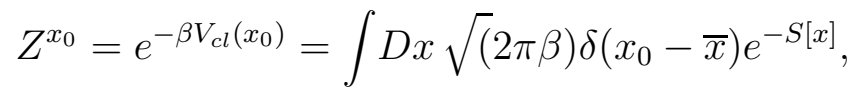

where $\bar{x}=\frac{\int d \tau x(\tau)}{\beta}$. For $T=0$ the classical effective potential coincides with the constraint effective potential [11].

The OE for the classical effective potential is generated by the application of the steepest-descent method to $Z^{x_{0}}$ (12) with the modified classical action (6). After shifting $x(t)$ by a constant $x_{0}$ and expanding the exponential 
into the Taylor series we have

$$
\begin{aligned}
& Z^{x_{0}}=e^{-\beta\left(\frac{\Omega^{2}}{2} x_{0}^{2}+\epsilon\left(\frac{1}{2}\left(m^{2}-\Omega^{2}\right) x_{0}^{2}+\lambda x_{0}^{4}\right)\right)} \\
& \times \quad \int D x^{\prime} \sqrt{(2 \pi \beta)} \delta\left(x_{0}-\bar{x}\right) e^{-\int_{0}^{\beta} \frac{1}{2} x^{\prime}(t)\left(-\partial^{2}+\Omega^{2}\right) x^{\prime}(t) d t} \\
& \times \quad\left[1-\epsilon\left(\frac{m^{2}+12 \lambda x_{0}^{2}-\Omega^{2}}{2} \int_{0}^{\beta} x^{\prime}(t)^{2} d t+\lambda x_{0} \int_{0}^{\beta} x^{\prime}(t)^{3} d t\right.\right. \\
& \left.\left.+\lambda \int_{0}^{\beta} x^{\prime 4}(t) d t\right)+\ldots\right] .
\end{aligned}
$$

After performing the Gaussian integrals, the local partition function and the classical effective potential can be calculated as series in $\epsilon$. The given order classical effective potential $V_{c l}^{n}\left(x_{0}\right)$ in the OE can be represented diagramatically, only one-particle irreducible diagrams are present, because by definition $x^{\prime}(t)$ has zero vacuum expectation value. Therefore, the set of diagrams is the same as in the case of the $\mathrm{OE}$ for the finite temperature effective potential, the only difference is in the propagator. In the case of the $\mathrm{OE}$ for the classical effective potential the propagator does not contain zero modes and equals

$$
G_{\beta}^{w z m}\left(t, t^{\prime}\right)=\frac{2}{\beta} \Sigma_{m=1}^{\infty} \exp \left[-i \omega_{m}\left(t-t^{\prime}\right)\right] \frac{1}{\omega_{m}^{2}+\Omega^{2}}=G_{\beta}\left(t, t^{\prime}\right)-\frac{1}{\beta \Omega^{2}}
$$

where $G_{\beta}\left(t, t^{\prime}\right)$ is the finite temperature propagator (8). Performing the integration over $x_{0}$ in (11) and calculating the free energy to the given order in $\epsilon$ we would obtain the result coincident with the $\mathrm{OE}$ for the finite temperature effective potential. However, a better approximation for the partition function can be obtained by reversing the order of operation: first to optimize the given order classical effective potential

$$
\frac{\delta V_{c l}^{(n)}}{\delta \Omega}=0
$$

and after to perform the integration over $x_{0}$ in (11) numerically. The first order of this approximation coincides with the Feynman-Kleinert (FK) variational determination of the classical effective potential [12, 13]. The OE gives a possibility to calculate corrections to the variational classical effective potential, improving the FK approximation for partition function in a 
systematic way. For alternative methods of calculating corrections to FK approximation see Refs. [10, 14].

5. We have studied the numerical results of the OE for the classical effective potential in the first and third order (to avoid complications with complex solutions of the second order). For $m^{2} \leq 0$ the quality of the approximation becomes worse for decreasing $\frac{m^{2}}{\lambda^{2 / 3}}$; the results for the quartic oscillator $\left(m^{2}=0\right)$ are compared with the exact free energy in Fig. 4. The first order results (FK1) are better than obtained from the value of the finite temperature effective potential at $\xi=0$ (OES1); however, the third order results (FK3) and (OES3) are very similar and agree well with the exact free energy calculated numerically. The differencies between the studied methods decrease to zero for $T=0$.

In the double-well case, the results of the OE for the classical effective potential are better than obtained from the lowest minimum (at $\xi \neq 0$ ) of the effective potential (OEN). The quality of the approximation improves for

increasing $\frac{\left|m^{2}\right|}{\lambda^{2 / 3}}$. For $m^{2}=-20$ the first order results (FK1) are indistinguishable from the exact results on the scale of Fig. 3. In Fig. 5 we compare the results of both methods for larger range of temperatures, the convergence in the FK approach is better than in the case of the OEN. However, one has to notice that the numerical calculations are much more complicated in the OE for the classical effective potential. Moreover, a generalisation of the FK approach to the true QFT (with an untrivial space dimension) is difficult, even in the lowest order.

\section{Acknowledgements}

This work has been supported partially by the Comitee for Scientific Research under Grant PB-2-0956-91-01. One of the autors (K.V.) is grateful to the members of the Institute of Physics (Białystok Branch of Warsaw University) and Soltan Institute for Nuclear Studies for the kind hospitality. 


\section{References}

[1] C. M. Bender and T.T. Wu, Phys. Rev. 184 (1969) 1231.

[2] R. Jackiw, Phys. Rev. D9 (1974) 3320.

[3] J. M. Cornwall, R. Jackiw, and E. Tomboulis, Phys. Rev. D10 (1974) 2428.

[4] A. Okopińska, Phys. Rev. D35 (1987) 1835.

[5] A. Okopińska, Ann.of Phys.(N.Y) in press.

[6] J.Schwinger, Phys. Rev.82 (1951) 664.

[7] A. Okopińska, Phys. Rev. D36 (1987) 2415.

[8] A. Duncan and H. F. Jones, Phys. Rev. D47 (1993) 2560.

[9] R. M. Quick and H. G. Miller, Phys.Rev. D31 (1985) 2683;

A. Okopińska, ibid D36 (1987) 1273.

[10] H. Kleinert, Phys. Lett. A 173 (1993) 332.

[11] R. Fukuda, E. Kyriakopoulos, Nucl. Phys. B85 (1975) 354; L. O'Raifeartaigh, A. Wipf and H. Yoneyama, Nucl. Phys. B271 (1986) 653.

[12] R. Giachetti and V. Tognetti, Phys. Rev. Lett. 55 (1985) 912, Int. Jour. Magn. Mater.54-57 (1986) 861.

[13] R. P. Feynman and H. Kleinert, Phys. Rev. A 34 (1986) 5080.

[14] J. Jaenicke and H. Kleinert, Phys. Lett. A 176 (1993) 409. 


\section{Figure captions}

Figure 1. The effective action to third order of the optimized expansion. The full line is an arbitrary propagator $G\left(t, t^{\prime}\right)$, the rule denotes the two-particle vertex $\Delta^{-1}\left(t, t^{\prime}\right)-G^{-1}\left(t, t^{\prime}\right)$.

Figure 2. The free energy $F$ of the quartic oscillator $\left(m^{2}=0\right)$, obtained as the value of the finite temperature effective potential at $\xi=0$ in first three orders (OES1, OES2, OES3) of the OE, plotted vs. the inverse temperature $1 / T$.

Figure 3. The free energy $F$ of the double-well oscillator $\left(m^{2}=-20\right)$, obtained in the $\mathrm{OE}$ as the value of the effective potential at $\xi=0$ (OES), and at $\xi \neq 0(\mathrm{OEN})$.

Figure 4. The free energy $F$ of the quartic oscillator $\left(m^{2}=0\right)$, calculated with the classical effective potential in first (FK1) and third (FK3) orders of the OE.

Figure 5. The free energy $F$ of the double-well oscillator $\left(m^{2}=-20\right)$, calculated with the classical effective potential in first (FK1) and third (FK3) orders of the $\mathrm{OE}$, compared with the value of the finite temperature effective potential at $\xi \neq 0$ (OEN1, OEN3). 
This figure "fig1-1.png" is available in "png" format from: http://arxiv.org/ps/hep-th/9311145v1 
This figure "fig2-1.png" is available in "png" format from: http://arxiv.org/ps/hep-th/9311145v1 
This figure "fig1-2.png" is available in "png" format from: http://arxiv.org/ps/hep-th/9311145v1 
This figure "fig2-2.png" is available in "png" format from: http://arxiv.org/ps/hep-th/9311145v1 
This figure "fig2-3.png" is available in "png" format from: http://arxiv.org/ps/hep-th/9311145v1 